\title{
Prevalence of Helicobacter pylori in benign gastric ulcers in a cohort of Sri Lankan patients
}

\author{
S Wijetunge ${ }^{1}$, R Kotakadeniya ${ }^{2}$, F Noordeen ${ }^{3}$, S M Buharideen ${ }^{1,3}$, B Samarasinghe ${ }^{2}$, A Dharmapala $^{2}$, \\ K B Galketiya ${ }^{2}$
}

(Index words: Helicobacter pylori, benign gastric ulcers, non-H. pylori gastric ulcers)

\begin{abstract}
Helicobacter pylori prevalence is decreasing globally and prevalence of non $H$. pylori gastric ulcers is increasing. The following study was conducted to assess the prevalence of $H$. pylori in benign gastric ulcers in a sample of Sri Lankan patients. This was a cross-sectional study of 59 dyspeptic patients with benign gastric ulcers. Multiple endoscopic gastric biopsies were obtained and histology, immunohistochemistry and polymerase chain reaction were performed for $H$. pylori detection. An immunochromatography assay was performed to detect blood anti $H$. pylori antibodies. Four $(6.8 \%)$ were positive for $H$. pylori. Therefore, it is likely that most benign gastric ulcers are of non- $H$. pylori aetiology.
\end{abstract}

Ceylon Medical Journal 2015; 60: 152-154

\section{Introduction}

With the description of a curved rod shaped bacterium (Helicobacter pylori) in a benign gastric ulcer in 1980s there was much enthusiasm to investigate the role of $H$. pylori in the pathogenesis of gastro-duodenal diseases [1]. Early studies have indicated 70 to $90 \%$ prevalence of $H$. pylori infection in benign gastric ulcers and 90 to $100 \%$ in duodenal ulcers [2]. However, more recent studies have indicated that the association between H. pylori infection and peptic ulcer disease is not as strong as indicated in previous literature [3-6]. The prevalence of $H$. pylori infection is coming down globally and this has been observed particularly in developed countries [7]. On the other hand, proportion of non-steroidal antiinflammatory drugs (NSAIDs) related peptic ulceration and non-NSAID, non- $H$. pylori ulcers are increasing in Western as well as in some developed Asian countries [36]. These observations indicate that the role of $H$. pylori in the pathogenesis of peptic ulcer disease is decreasing.
There are only a few studies on aetiological association of benign gastric ulcers from Sri Lanka. Fernando et al. reported a $H$. pylori prevalence of $59 \%$ and $58 \%$ in duodenal and gastric ulcers respectively using only CLO test, and Waidyarathne et.al reported a prevalence of $51.8 \%$ in gastric ulcers using only histology alone $[8,9]$. We conducted the present study to assess the prevalence of $H$. pylori infection in benign gastric ulcers in a cohort of Sri Lankan patients, using histology, immunohistochemistry, polymerase chain reaction and serology as $H$. pylori detection methods.

\section{Methods}

This was a cross-sectional study of dyspeptic patients undergoing upper gastrointestinal endoscopy (UGIE) from March 2012 to August 2013, at the Teaching Hospital, Peradeniya, Sri Lanka. Patients who were detected to have benign gastric ulcers, confirmed with histology, were selected for analysis. Mucosal breaches more than 3 $\mathrm{mm}$ in diameter with some depth were regarded as ulcers and superficial smaller breaches which were classified as erosions were excluded [10]. Multiple endoscopic biopsies were obtained from the ulcer edge, antrum, insicura angularis and the body from each patient. Histology based haematoxylin and eosin stain and toluidine blue stain, immunohistochemistry and polymerase chain reaction (PCR) were performed on gastric biopsies of every patient to detect $H$. pylori organisms. Venous blood was drawn from each patient to assess anti $H$. pylori antibodies. Positivity with any one of the test methods was regarded as evidence of $H$. pylori infection.

Biopsies for histology and immunohistochemistry were collected in $10 \%$ formaldehyde and the biopsy for PCR was collected fresh and stored at $-80^{\circ} \mathrm{C}$. Immunohistochemical staining was performed using indirect immunoperoxidase method (DAKO B0471 anti $H$. pylori antibodies) on formalin fixed paraffin embedded

Departments of ${ }^{1}$ Pathology, ${ }^{2}$ Surgery and ${ }^{3}$ Microbiology, Faculty of Medicine, University of Peradeniya, Sri Lanka.

Correspondence: SW, e-mail: <suwijetunge@gmail.com>. Received 24 February and revised version accepted 18 April 2015. and reproduction in any medium, provided the original author and source are credited. 
tissue. PCR was performed using primer HP1/HP2 for 16SrRNA locus. Serum anti H. pylori antibodies were assessed using a qualitative immunochromatography based assay (SD BIOLINE). This method collectively detects anti $H$. pylori antibodies and cannot differentiate the antibody sub- types; the test has a sensitivity of $95.5 \%$ and specificity of $89.6 \%$, according to the manufacturer. Accordingly, this test was used as a screening method to detect anti $H$. pylori antibodies.

As quality control measures, positive controls were run with immunohistochemistry, PCR and immunochromatography methods and for PCR, in addition, tests were performed in duplicates.

Approval for the study was obtained from the Ethical Review Committee of Faculty of Medicine, University of Peradeniya.

\section{Results}

A total of 656 dyspeptic patients underwent UGIE during the study period and of them $59(8.4 \%)$ had benign gastric ulcers. The mean age of the patients with ulcers was $62 \pm 14.2$ years (range 22 to 89 years) and $35(59.3 \%$ ) were males. Fifty six ulcers were in the antrum and the rest in the proximal stomach. Almost all the ulcers were less than $1 \mathrm{~cm}$ in diameter and none exceeded $2 \mathrm{~cm}$. Twenty nine ulcers were associated with erosions elsewhere in the gastric mucosa and three were associated with duodenal ulcers and two with duodenal erosions. Twenty two had a history of haematemesis, melaena or both.

One showed positivity for $H$. pylori with all the test methods used, and three were positive with PCR only. Overall, four cases $(6.8 \%)$ showed positivity for $H$. pylori with at least one of the test methods used. All $H$. pylori positive ulcers were present in the gastric antrum (two in the pylorus, one in the incissura and one in the rest of the antrum) and all had associated erosions. Duodenum was unremarkable in all cases. The mean age of $H$. pylori positive cases was $69.8 \pm 19$ years and in $H$. pylori negative cases $61.4 \pm 13.8$ years (the difference was not statistically significant). Three patients with $H$ pylori positive gastric ulcers were men.

\section{Discussion}

In the studied population with gastric ulcers, only $6.8 \%(\mathrm{n}=4)$ had evidence of $H$. pylori infection and the majority $(93.2 \%$ ) had no evidence of $H$. pylori infection with any of the test methods used. Seroprevalence of anti-H.pylori antibodies was $1.7 \%$, which indicates low exposure rate to $H$. pylori in the study population. Furthermore, this very low seroprevalence excludes the possibility of false negative results due to previous exposure to antibiotics.

Western literature shows that with the declining role of $H$. pylori in the pathogenesis of gastric ulcers, drug related causes, especially NSAIDs and low dose aspirin have gained importance [3-6]. Furthermore, prevalence of non NSAID non $H$. pylori gastric ulcers is known to be increasing and the aetiology of this group of ulcers is still not clear [3-6]. The role of bile reflux in the pathogenesis of these ulcers is not known because of the difficulty in assessing the degree of bile reflux. Although diseases such as Zollinger-Ellison syndrome, hyperparathyroidism and gastric Crohn disease are known to cause gastric ulcers, these diseases are not common enough to explain the relatively high prevalence of non-NSAID non- $H$. pylori gastric ulcers. Psychological and physical stress, cigarette smoking and genetic predisposition are other contributory factors of gastric ulcers.

Under-recognition of non- $H$. pylori causes of gastric ulcers could lead to unnecessary treatment of patients with $H$. pylori eradication therapy and ulcer recurrence. Therefore, it is time to shift our focus from H. pylori and consider other possible causes for gastric ulcers.

\section{Funding}

Financial assistance was from the National Science Foundation of Sri Lanka (NSF Grant No. RG/2011/ HS/11) and University of Peradeniya (University Grant No. RG/2012/34/M).

\section{Acknowledgements}

Authors thank Mrs. T.R.D.S.K. Tennakoon, Mrs. S.M. Wickramasingha and Mr. A.M.S.B. Abeykoon for technical assistance.

\section{Conflicts of interests}

There are no conflicts of interest.

\section{References}

1. Marshall BJ, Warren JR. Unidentified curved bacilli in the stomach of patients with gastritis and peptic ulceration. Lancet 1984; 1: 1311-5.

2. Kuipers EJ, Thijs JC, Festen HP. The prevalence of Helicobacter pylori in peptic ulcer disease. Aliment Pharmacol Ther 1995; 9: 59-69.

3. Laine L, Hopkins RJ, Girardi LS. Has the impact of Helicobacter pylori therapy on ulcer recurrence in the United States been overstated? A meta-analysis of rigorously designed trials. Am J Gastroenterol 1998; 93: 1409-15.

4. Yuan Y, Padol IT, Hunt RH. Peptic ulcer disease today. Nat Clin Pract Gastroenterol Hepatol 2006; 3: 80-9.

5. Higham J, Kang JY, Majeed A. Recent trends in admissions and mortality due to peptic ulcer in England: increasing frequency of haemorrhage among older subjects. Gut 2002; 50: 460-4.

6. Iijima K, Kanno T, Koike T, Shimosegawa T. Helicobacter pylori-negative, non-steroidal anti-inflammatory drug negative idiopathic ulcers in Asia. World J Gastroenterol 2014; 20: 706-13. 
7. Ford AC, Axon ATR. Epidemiology of Helicobacter pylori infection and Public Health Implications. Helicobacter 2010; 15: 1-6.

8. Fernando F. Helicobacter pylori and peptic ulcer disease in Sri Lanka. Ceylon Med J 1992; 37: 15-7.

9. Waidyarathne E, Mudduwa L, Lekamwasam S, Lekamwasam V, Gunawardhana H. Prevalence of Helicobacter pylori infection determined by histology in patients with different upper gastrointestinal diseases. Ceylon Med J 2010; 55: $68-9$.

10. Tamura A, Murakami K, Kadota J. Prevalence of gastroduodenal ulcers/erosions in patients taking low dose aspirin with either $15 \mathrm{mg}$ /day of lansoprazole or $40 \mathrm{mg} /$ day famotidine: the OITA-GF study 2. BMC Res Notes 2013; 6: 116 